\title{
Abdomino Pelvic Tuberculosis Versus Advanced Ovarian Malignancy: A Diagnostic Dilemma
}

\author{
Serajun Noor ${ }^{1 *}$ \\ Nurun Nahar ${ }^{1}$ \\ Afroza Bilkis ${ }^{1}$ \\ Taniza Jabin ${ }^{2}$ \\ 'Department of Obstetrics \& Gynecology \\ Chittagong Medical College \\ Chittagong, Bangladesh. \\ ${ }^{2}$ Department of Medicine \\ Chattagram Metropoliton Haspatal \\ Chittagong, Bangladesh.
}

${ }^{*}$ Correspondence to:

\section{Dr. Serajun Noor}

Associate Professor

Department of Obstetrics \& Gynecology

Chittagong Medical College

Chittagong, Bangladesh.

Mobile : +8801619310294

Email : noorserajun@yahoo.com

www.banglajol.info/index.php/CMOSHMCJ

\begin{abstract}
Pelvic tuberculosis a common infectious cause of human death remains a global health problem, primarily in developing countries .Diagnosis of extra-pulmonary tuberculosis is challenging as it is rarely pinpointed by clinical symptoms, laboratory and radiological findings of low specificity. Pelvic tuberculosis should be considered in young women presented with pelvic mass, ascites and elevated serum CA 125. A series of fourteen cases between the 20 to 70 years are being reported having spectrum of clinical feature and creating diagnostic dilemma. The final diagnosis was made by histopathology after FNAC, laparoscopy or laparotomy are reviewed here. So the aim was formulation of high degree of suspicion aided by intensive investigation for timely diagnosis of genital tuberculosis, to see the response of antituberculer therapy and avoid unnecessary extended surgery.
\end{abstract}

Key ward: APTB; Advanced ovarian malignancy; Serum CA 125; Ascites.

\section{INTRODUCTION}

A female abdomino-pelvic tuberculosis a global health problem, is a disease of young adult and its occurrence in post-menopausal women is believed to be rare, $1 \%$ of post menopausal bleeding ${ }^{1}$. Genitourinary tuberculosis accounts for about $15 \%$ of all extra pulmonary tuberculosis and after lung is the most common site ${ }^{2}$. Pelvic TB is common in female gender so gynaecologist need to be aware that tuberculosis may present in an atypical manner ${ }^{3}$.

Among the genital disease Genital Tuberculosis (GTB) is very baffling because of its various presentations. Genital TB frequently present without symptom and diagnosis require a high index of suspicion. The disease can mimic many conditions, including bowel disease, malignancy and other infectious disease. Serum CA 125 is a soluble glycoprotein which is increased in chronic infection of mesothelial cells of peritoneum, pleura, and pericardium. It also increased in many gynecological and non gynecological conditions as well as $80 \%$ patients with ovarian malignancy ${ }^{4}$. It is a valuable parameter to measure the pelvic TB activity ${ }^{5}$. The clinical picture of GTB is so variable that the single symptom, sign or investigation may not be suggestive. Pelvic TB may present with pelvic pain, infertility, poor general health or menstrual disturbance. Adhesion between fallopian tube, ovaries, omentum, intestine, liver and diaphragm in the form of abdominal mass can also present. Pelvic TB presenting as abdominal lump with raised CA 125 and ascities can be a differential diagnosis of advanced ovarian malignancy or disseminated peritoneal Carcinomatosis. This can lead to unnecessary laparotomy and radical surgery where as pelvic TB respond well to medical treatment.

Different specific test -isolation of organism AFB staining, culture, Gene-X part test, antituberculer antibody, Spoloigotyping moleculer methodology[6], biopsyhistopathology and nonspecific test-CBC, X -ray chest, Adenine-diaminase of ascitic fluid, tuberculin test can be done in multiple phase. 
Ultrasonography, CT scan or MRI of pelvis, serology can differentiate abdomino- pelvic tuberculosis from malignancy but final diagnosis is revealed by FNAC or laparoscopy or laparotomy followed by biopsy and histopathology. Abdominal and pelvic CT indicates dense, granuler and septate ascites and swelling of bowel wall. Laparoscopy guided biopsy seems to be of surmount and safe method to provide diagnosis of pelvic TB. If laparoscopy seems to be not feasible specially in suspected dry or fibroadhesive tuberculosis laparotomy should be performed. Though absolute diagnosis can not be made from characteristic feature in HCG or laparoscopy , laperoscopy is a valuable procedure for obtaining tissue and histopathological examination.

If no malignancy is detected and diagnosis of pelvic TB is confirmed extended surgery is avoided. As tuberculosis require only conservative management it has been suggestive that in case of abdomino- pelvic masses with ascities and high serum CA 125 should always be suspicious of pelvic tuberculosis. Serology, culture and radiology if negative for TB, laparoscopy or laparotomy combined with biopsy should be performed to confirm the diagnosis. This will prevent unnecessary extended surgery. Moreover serum CA 125 can be used to monitor the response of disease to anti TB therapy.

In this paper 14 cases with diagnostic dilemma of preoperative differential diagnosis between abdomino pelvic tuberculosis and advanced ovarian malignancy or peritoneal carcinomatosis were analysed. The aim of study was formulation of high degree suspiscion criteria, inclusion and exclusion criteria aided by intensive investigation for early diagnosis of abdomino pelvic tuberculosis . Thereby to have good prognosis, to see response of anti TB therapy and to avoid unnecessary extended surgery.

\section{MATERIALS \& METHODS}

In this descriptive study (Case series) 14 patients were recruited in department of gynaecology (Admitted or reffered) from January 2012 to April 2015 .

Patients demographic profile, clinical features with associated medical illness, past medical history, menstrual status and family history were evaluated. Data about routine clinical $(\mathrm{G} / \mathrm{E}$, $\mathrm{P} / \mathrm{A}, \mathrm{P} / \mathrm{V}$ ) examinations, routine biochemical tests, Purified Protein Derivative (PPD) status, Serum CA 125, ascitic fluid study, AFB staining and culture, PCR, ADA, chest X-ray, Gene$\mathrm{X}$-part test, abdominal USG,CT results as well as operative (laparoscopy or laparotomy) findings and histopathology report were recorded.

\section{RESULTS}

Though abdomino- pelvic tuberculosis is a disease young adult, $10(71 \%)$ patients were post menopausal and 13

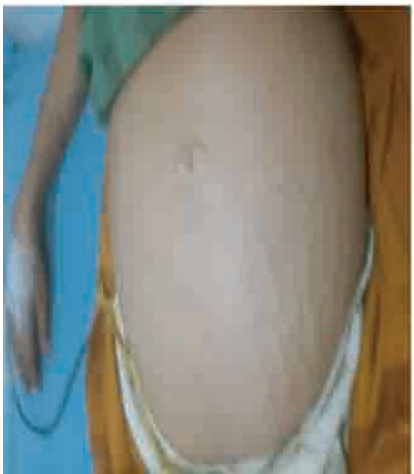

Figure $1:$ Pelvic TB with ascites

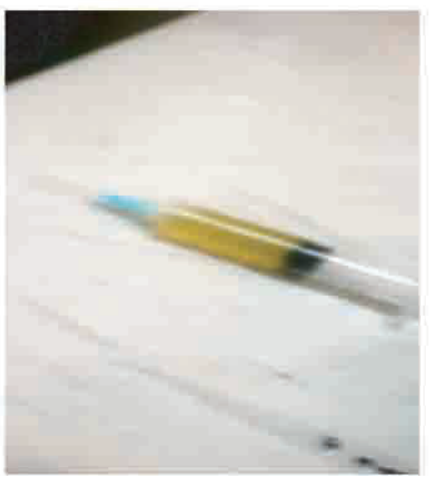

Figure 2 : Ascitic fluid in TB
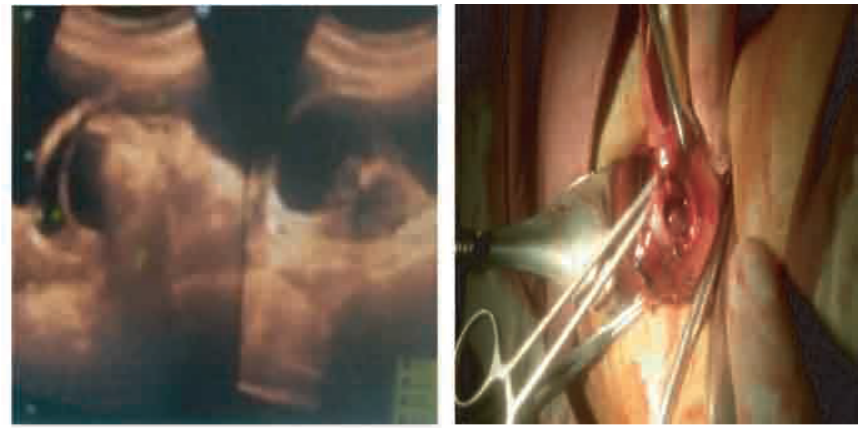

Figure 3 : Septated ascites in USG

Figure 4 : Ectopic pregnancy inTB

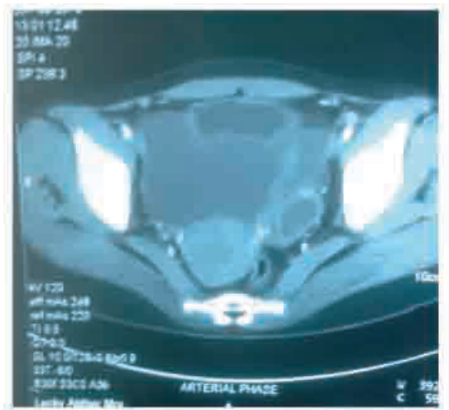

Figure 5 : Pelvic mass (TB) in CT

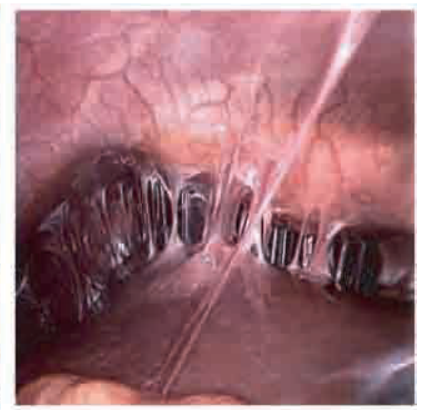

Figure 6 : "Violin string" (Laparoscopy)

from low socioeconomic status . Median age were 25-70 years. Mean duration of symptom for 4 month to 6 years. The major clinical manifestations were abdominal pain, weight loss, abdominal distension, diffuse and solid abdominal mass with ill defined margin in 10 patients and only ascites without any palpable mass in 4 patients. Huge ascites were detected clinically in 12 patients while sonologically in 2 patients. Serum CA 125 was raised in all 14 patients $(400-5000 \mathrm{U} / \mathrm{ml}$, normal $<35 \mathrm{U} / \mathrm{ml}) . \mathrm{Hb} \%$ was around $(8-10 \mathrm{gm} / \mathrm{dl})$. Complete blood count shows lymphocyte predominance, ESR raised in all 14 patients $\left(40-120 \mathrm{~mm}\right.$ in $1^{\text {st }}$ hour) and PPD $(+)$ ve in one cases. Abdominal paracentesis performed in 12 clinically detected ascites and discovered straw colored fluid, lymphocyte predominance (50-88\%) with negative cytology for AFB, malignant cells and bacteriological culture.

USG show multi septated ascites in all 14 patients and USG guided aspiration of ascitic fluid done in 4 patients. Ascitic fluid Adenosine Di Aminase (ADA) activity has been proposed as an useful diagnostic test for abdominal tuberculosis and were raised in all 14 patients $(50-120 \mathrm{IU} / \mathrm{L})$ (normal $<32 \mathrm{IU} / \mathrm{L})$. $\mathrm{PCR}+\mathrm{ve}$ in only 4 patient range $(120$ to $200 \mathrm{mg} / \mathrm{L}$, normal $<5 \mathrm{mg}$ ). USG guided FNAC from abdominal mass done in 8 patients, CT guided FNAC in 2 patients and diagnosed TB only in 2 cases but excluded malignancy by benign nature in 12 .

Diagnostic laparotomy was done in 9 cases with differential diagnosis of ovarian malignancy and laparoscopy in 1 case. 5 patients with all laboratory test negative for tuberculosis were advised for diagnostic anti tubercular therapy for 1 month. Among them a 25 years lady inspite of disappearance of fever and improvement of appetite discontinued drug after 10 days. 
Considering her age and doughy abdomen (Suspected adhesion), a diagnostic laperotomy was done with a differential diagnosis of ovarian malignancy and the findings were in favour of fibroadhesive type of tuberculosis. Other 4 patients improved clinically with antituberculer drug, serum CA 125 reduced, ascites and abdominal lump disappeared.

Conservative surgery followed by anti tubercular therapy were done salphingectomy in 1 patient due to TB with chronic ectopic pregnancy, bilateral salphingectomy for bilateral hydrosalpnix, excision of encysted tubercular abscess in 1 and presence menstrual abnormality promted TAH with BLSO in 1 patient aged 50 years although3- 6 weeks preoperative antituberculer therapy would have been ideal.

\section{DISCUSSION}

An abdomino pelvic tuberculosis require conservative anti tubercular treatment while ovarian malignancy demands extended surgery, chemotherapy even radiotherapy. However diagnostic dilemma of abdomino pelvic tuberculosis presents the technical hindrance for proper therapies in these patients when Extra Pulmonary TB (EPTB) is suspected. Usually women with EPTB were younger than women with advanced ovarian malignancy peak age (20-40 yrs) and rare in post menopausal women. But in our study 10(71\%) cases were post menopausal (55-70yrs). This advanced age may be due to absence of constitutional symptoms like fever, sweating, anorexia, weight loss or presence of nonspecific symptoms ascites, mass mimicking host of other conditions, delaying diagnosis. Use of combination of radiologic, microbiologic, histopathologic, endoscopic and moleculer technology contribute to delayed diagnosis.More over tuberculosis may remain for 10 to 18 years ${ }^{6}$. Patients with chronic pelvic pain, ascites ,fever, weight loss and pelvic mass favour pelvic $\mathrm{TB}^{6}$. Laperotomy or laparoscopic criteria for diagnosis of abdominal TB are free peritoneal fluid, peritonitis, perisalphingitis, fibrosis in tubal anti-mesenteric border, caseating flimsy adhesion with uterus, peri uteritis, encysted fluid, multiseptated ascites and uterine synaechia.

In this study ascites was present in 14 patients (100\%), 12 (85\%) diagnosed clinically and $2(15 \%)$ sonologically. Munef et al in 2001 reported ascites in $61 \%$ patients. Roya Nasiri et al reported 15 cases of pelvic TB with ascites ${ }^{7}$.

Tubercular ascetic fluid is straw colored usually exudative with high protein content, lymphocytes predominant but rarely retrieve Tubercle bacilli in AFB staining, bacteriology and culture because extra-pulmonary TB is paucibacillary.

Low grade fever and weight loss though a consistent finding of $\mathrm{TB}$, it is uncommon in EPTB. In our series only 3 patients (30\%) reported weight loss and 1 patient $(7 \%)$ low grade fever. In Hatami's study on 52 patients of pelvic TB no patient suffered from fever ${ }^{8}$. Sheets et al in a series of 19 cases reported weight loss and fever in $40 \%$ patients while ( $\mathrm{Li} \mathrm{XJ}$ et al) reported fever in $70 \%$ patients 9 . Abdominal distention moderate to severe degree was present in all 14 patients $(100 \%)$ as a consistent findings similar to other studies ${ }^{10,11}$.
There is usually no clinical or radiological evidence of primary $\mathrm{TB}$, as primary focus in the lungs usually heals completely. Chest X ray report in all 14 patients agreed with other studies and do not reveal any sign of active or old $\mathrm{TB}^{12}$. Negative chest radiograph cannot exclude the presence of EPTB. Only 37\% patients (Seeled had history of TB,7\% cases in (Unay) but negative $\mathrm{X}$ ray. In our case series only 1 patient had $\mathrm{H} / \mathrm{O}$ TB (7\%)and another one $\mathrm{F} / \mathrm{H} / \mathrm{O}$ TB while remaining 12 had no evidence of positive personal or family history tuberculosis,

Someet reported $<30 \% \mathrm{~F} / \mathrm{H} / \mathrm{O} \mathrm{TB}, 5 \%$ in U. Onkey et al $2004^{13,14}$

There are lots of study reported raised CA 125 with or without ascites and pelvic mass mimicking advanced ovarian malignancy (Thanker et al) ${ }^{15}$. All 14 patients $(100 \%)$ in this series with elevated CA 125 were admitted in gynecology department or referred from medicine department, evaluated as advanced ovarian malignancy and ultimately diagnosed as abdomino- pelvic tuberculosis. Yilmaz et al in their case control study on 96 subjects showed that serum CA125 in pelvic TB with a mean level of $109.7 \mathrm{U} / \mathrm{ml}$ with a sensitivity and specificity of $97.5 \%$ and $100 \%$ respectively at a $31 \mathrm{U} / \mathrm{ml}$ cut off point ${ }^{16}$.

Pre operative paracentesis were unhelpful because the bacteriology, culture, cytology(14,100\%) and PCR(10,71\%) study of ascitic fluid yield negative result similar to earlier reports (Rozati et al) ${ }^{17}$.

PCR of ascitic fluid obtained by USG/CT guided fine needle aspiration is a investigation of choice in suspiscious APTB, it is positive in $60 \%$ cases of ascites in TB and only $19 \%$ patient in this series. False positive PCR with negative clinical findings may be due to early disease with low number of bacilli or latent infection which is picked up by PCR when women are still asymptomatic and before the structural damage to the fallopian tubes has taken place. However as there is no gold standard tool to diagnose APTB and to compare PCR one should be cautious of false positivity byway of contamination, dead bacilli, previous infected or asymptomatic TB at another sites. False negative $(10,71 \%)$ results in PCR may be due to paucibacillary nature of the specimen or portion of the specimen taken for PCR may not have any Mycobacterium Tubercle bacilli. Contamination of ascitic fluid by blood could possibly explain false negative result in this study ${ }^{20}$. However addition of PCR to culture ,bacteriology can increase the efficacy ${ }^{18}$. Ascetic fluid ADA activity is a useful diagnostic test for abdominopelvic tuberculosis and in countries with high incidence of TB the procedure can used as an screening test ${ }^{19}$. In our case series ADA was raised in all 14 patients (50-120 U/L). ADA level $>32 \mathrm{U} / \mathrm{L}$ with $100 \%$ sensitivity and $92-100 \%$ specificity can obviate the need for more invasive and expensive test ${ }^{19}$.

Low incidence of culture, cytology and PCR positivity could be due to paucibacillary nature \& substantial number of tuberculous lesion of the genital tract are bacteriologically mute. And presence of bacteriostatic substances inhibit the growth of bacillii ${ }^{20}$. 
USG and CT guided FNAC followed by histology of TB lesion can provide characteristic feature. CT can diagnose TB in $75 \%$ cases. But due to secondary nature of genital TB, the infecting organisms are sparse in number, sample may not represent the infected area and the infected site can be easily missed. CT can easily miss a lesion of $4-5 \mathrm{~mm}$.Presence of moving septum in USG, thick endometrium in absence of post manupausal bleeding and normal omentum in $\mathrm{CT}$ scan favour TB than ovarian malignancy ${ }^{21}$. If pelvic TB suspected histological examination of premenstrual endothelial curettage may yield granuloma and reported APTB in 50 to $70 \%$ cases, mimicking advanced ovarian malignancy. Though endometrial curettage not done in any 14 patient due to absence of menstrual abnormality positive histology on endometrial biopsy was seen in $3.31 \%$ patients Madhu etal [20,] similar to Manjari et $\mathrm{al}^{20}$. The lower rate may be due to cyclical shedding of endometrium and absence of reinfection in every cycle, scany specimen and blood flow ${ }^{20}$. Usually dry TB without ascites show endometrial involvement, adenexal masses, loculated fluid and adhesion. Patients improvement are evidenced by anti tubercular therapy evidenced by decrease in ascites, serum CA 125 level and resolution of abdominal distention and mass ${ }^{22}$.

Laparoscopic peritoneal biopsy is a rapid and safe method of accurate diagnosis of APTB in $97 \%$ cases as $<1 \mathrm{~cm}$ tubercle can be identified. The sensitivity of gross laparoscopic appearance in diagnosing peritoneal TB is almost $100 \%{ }^{23}$. Direct biopsy can also be taken with very low risk. Laparoscopic findings of small yellow small granules with homogenous distribution, violin string fibrogenous tissue, diffusely distributed uniform peritoneal nodule 4-5mm, extensive adhesion, matted loop of bowel, thickened rolled up omentum like omental cake are typical of APTB. APTB can present in 3 pattern- wet type with ascites, dry type with adhesion and encysted locules with localized swelling. Fibrotic type with omental thickening abdominal mass, peritoneum studed with multiple yellow tubercles. It is thickened, hyperemic with loss of shiny texture. Wadia criteria for diagnosis of APTB are free peritoneal fluid, periuteritis, blue uterus, perisalphingitis TB lossed like caseating flimsy adhesion, encysted fulid and Uterine synechia ${ }^{23}$.

In our series diagnostic laparoscopy was done in a 25 years old lady with differential diagnosis of tubo-ovarian mass. Laparoscopy revealed moderate amount of straw colored ascitic fluid, violin string adhesion and a mass due to flimsy adhesion between left fallopian tube, ovary and omentum, having caseous material within the lump.
Diagnosis of APTB confirmed after histology and antitubercular drug was started. She subsequently conceived after antituberculer therapy \& with ovulation inducing drugs along with Prednisolone. Though infertility is common in pelvic TB, a stray cases with no gross pathology can become pregnant. Wadia reported repeat pregnancy in $5 \%$ cases, it be $30 \%$ with newer antibiotics and prednisolone in a doses of $20 \mathrm{mg}$ per day ${ }^{23}$. The pregnancy rate jumped to over $60 \%$ with larger doses of steroids and newer antituberculer drugs. Addition of ciprofloxacin gave result nearing $75 \%$ and addition of gamma globulin raise the success to almost $80 \%$. Wadia also reported increased incidence of ectopic pregnancy due to TB in india.

Diagnostic laparotomy seems to be comparatively safe in dry \& fibroadhesive type of APTB ${ }^{23}$. In our series 9 patients among 14 had diagnostic laparotomy with only biopsy in 6 cases, bilateral salphingectomy in 1 (hydrosalphinx), unilateral salphingectomy in 1 (Chronic ectopic), excision of encysted mass $(30 / 20 \mathrm{~cm})$ and drainage of caseous material in 1 case. Diagnosis was confirmed after histology. One patient with fever (100.4), sonologically detected ascites \& TO mass suspicious of TB, clinically \& on Lab findings, responded well to diagnostic anti TB therapy. Her lump size reduced \& ascites disappeared but patient herself stopped anti TB drugs after 11 days of therapy. Then considering her age, diagnostic laparotomy done \& reaveled fibro-adhesive TB, confirmed by histology. Biglin et al reported 10 patients with peritoneal TB who were operated for suspected advanced ovarian malignancy ${ }^{23}$.

High false negative result and small sample size are the limitation of the study.

\section{CONCLUSION}

Pelvic peritoneal TB should always be considersd in a patient with adenexal mass and ascites, especially in poor economic and health condition. Family history and fever are important factors differentiating pelvic TB from advanced ovarian cancer. Medical and conservative surgery is the aim of treatment. In depth knowledge and diagnostic means are needed to have earlier diagnosis, effective medical and surgical therapies with preservation of reproductive capability.

\section{DISCLOSURE}

All the authors declared no competing interest. 


\section{REFERENCES}

1. Baratnur S,RamkumarV,KudvaR:Abdominopelvic tuberculosis Masquarding ovarian malignancy-Histology key to management. Indian Journal of MDS. 2014;3(1).

2. Saba Imtiaz,Neelam Siddiqui, Murtaza Ahmed, and Amna Jahan .Pelvic-peritoneal Tuberculosis Mimicking Ovarian Cancer.Journal of College of Physicians and Surgeons Pakistan. 2012;22(2):113-115.

3. Mondal S,K.D.K.Tapan,N.R.Dipanwita et al .Histologic Analysis of Female T uberculosis with clinical correlation: A Fifteen years study in a Tertiary Hospital of India. Journal of bcrs.org. 2012; 1(1).

4. S.Chhabra,K.Shaharan and D.Pohane.Pelvic tuberculosis continues to be a disease of diagnostic dielemma- case series. Indian Journal Tuber. 2010;57: 90-94.

5. Barutcu O,Erel HE, Saygili E, Yildirim T,TorunD.Abdominopelvic tuberculosis simulating disseminated ovarian carcinoma with elevated CA 125 level: report of two cases.Abdom Imaging. 2002;27:465-470.

6. Jana N, Mukhopadhyay S,Dhali GK.Pelvic tuberculos .J of Obsted Gynae of India. 2007;27:226-227.

7. Penna L,Manyonda I, Amias A-Intra-abdominal milliary tuberculosis presenting as advanced ovarian carcinoma with raised CA125 Gynaecol Obsted Invest. 2001;51(4):277-279.

8. Hatami M.Tuberculosis of the female genitaltract in Iran.Archives of Iranian Medicine. 2005;8:32-35.

9. Li XJ, Wu LY, Li XG, Sun YC. Analysis of 20 cases of pel vic tuberculosis initially suspected of ovarian carcinoma.Zhonghua Jie He He Hu Xi Za Zhi. 2003;26:462-464

10. Sinha S,Chidamberan -Pillai S.Pelvic tuberculosis: an uncommongynaecological problem presenting as ovarian mass.BJOG $2000 ; 107 ; 139-140$

11. Hatami M.Tuberculosis of the female genitaltract in Iran.Archives of Iranian Medicine. 2005;8:32-35.

12. Sheets E E,Smith RN . Case report-A 31 year old woman with Plural effusion,ascites and persistant fever spike.N Engl Med. $2008 ; 338: 248-254$.

14. Hussain SF, Camilleri P.Elevation of tumour marker CA125 in Serum and body fluids: interpret with caution. Indian J Med Res. 2007; 125: 25-30.

15. Thankur V Mukharjee U, Kumar K.Elevated serum cancer antigen125 levelsin advancedabdoinal tuberculosis.Med Oncol. 2001;18:289-291

16. Lantheaume S, Soler S, Issartel B,Isch JF,Lacassin F,Rougier Y,et al .Peritoneal tuberculosis simulating advanced ovarian cancer: a case report .Gynecol Obstet Fertil. 2003;31:624-626.

17. Manidakis LG ,Angelakis E, Sifakis S,Stefanaki P, Kalogeraki A,Manidaki A,et al .Genital tuberculosis can present as disseminated ovarian carcinoma with ascites and raised CA 125 : A case report .Gynecol Obsted Invest. 2001;51:277-279.

18. Nebhani M,Boumzgou K,Brams S,Laghzaoui M,EI Attar H,Bouhya S,et al. Pelvic tuberculosis mimicking bilateral Ovarian tumour : A case report J Gynaecol Obstet Biol Reprod. 2004;33:145-147.

19. Koc S,BeydilliG,Tulunay G,Ocalan R,Boran N,Ozgul N,et al .Peritoneal tuberculosis mimicking advanced ovarian cancer: A Retrospective review of 22 cases. Gyncol Oncol. 2006;103:565-569.

20. R.B.P.Thangappah,C.NParamasivan \& Sujatha Narayanan.Evaluating PCR,culture \& histopathology in the Diagnosis of female genital tuberculosis. 2011;134:40-46.

21. PiuraB. RabinovichA,Leron E, Yanai-InbarI, Mazor M.Pertonealtuberculosis mimicking carcinoma with ascites and elevated serum CA 125: Case report and review of literature.Eur J Gynaecol Oncol. 2002;23:120-122.

22. Yilmaz A, Ece F, Bayramgurler B, Akkaya e,Baran R.The value of Ca 125 in the evaluation tuberculosis activity.Respir Med 2001;95:666-669.

23. Bashar A.Abdul Hasan,Anees K.Nile,Enas Adam Abdul rasul Al Kazzaly.Role Surgery and Laperoscopy in Management of Abdominopelvic Tuberculosis. The Iraqi post graduate medical Journal. 2011;10. 